\title{
Event Based Clustering Localized Energy Efficient Ant Colony Optimization for Performance Enhancement of Wireless Sensor Network
}

\author{
Shankar D. Chavan \\ Department of E\&TC \\ Sinhgad College of Engineering \\ Pune, Maharashtra, India \\ sdchavan27@rediffmail.com
}

\author{
Anju V. Kulkarni \\ Dr. D. Y. Patil Institute of Technology \\ Pune, Maharashtra, India \\ anju_k64@yahoo.co.in
}

\begin{abstract}
The main challenge of a wireless sensor network (WSN) in disaster situations is to discover efficient routing, to improve quality of service $(\mathrm{QoS})$ and to reduce energy consumption. Location awareness of nodes is also useful or even necessary. Without knowing the position of sensor nodes, collected data is insignificant. Ant colony optimization (ACO) is a unique form of optimization method, which is highly suitable for adaptive routing and guaranteed packet delivery. The primary drawbacks of ACO are data flooding, huge overhead of control messages and long convergence time. These drawbacks are overcome by considering location information of sensor nodes. An event-based clustering localized energy efficient ant colony optimization (EBC LEE-ACO) algorithm is proposed to enhance the performance of WSN. The main focus of the proposed algorithm is to improve QoS and minimize the network energy consumption by cluster formation and selecting the optimal path based on the biological inspired routing-ACO and location information of nodes. In clustering, data is aggregated and sent to the sink (base station) through cluster head $(\mathrm{CH})$ which reduces overheads. EBC_LEE-ACO is a scalable and energy efficient reactive routing algorithm which improves QoS, lifetime and minimizes energy consummation of WSN as compared to other routing algorithms like AODV, ACO, ACO using RSSI. The proposed algorithm reduces energy consumption by approximately $7 \%$, in addition to improvement in throughput, packet delivery ratio and increase in packet drop which has been observed in comparison with other algorithms, i.e. autonomous localization based eligible energetic Path with Ant Colony optimization (ALEEP with ACO) of the network. Use of IEEE 802.11 standard in proposed work increased packet drop.
\end{abstract}

Keywords-ACO; ALEEP; AODV; CH; clustering; EBC; LEE; QoS; RSSI; WSN

\section{INTRODUCTION}

WSN consists of a number of sensor nodes. These nodes are small in size and have low power capacity. These multifunctional nodes are deployed in a particular area for data gathering purposes. The sensor nodes (SN) pass the data to the sink node using multiple hops as shown in Figure 1. The requirement in disaster situations is that the sensor nodes should perform the communication without failure for long duration. A traditional routing algorithm does not consider the location information and thus cannot be used in disaster areas where stable communication is important. Selection of routing algorithm is one of the major problems of sensor network to be solved [1-3]. The main goals of WSN routing are to improve QoS and network lifetime and to reduce connectivity failure.

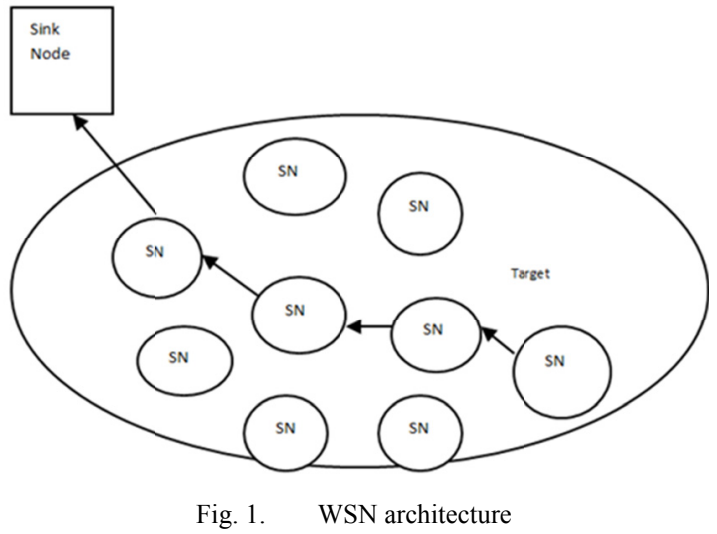

Therefore, an event based clustering localized energy efficient ant colony optimization (EBC_LEE-ACO) algorithm is proposed. The main focus of the proposed algorithm is to enhance QoS, to minimize network energy consumption by cluster formation and to select the optimal path based on the biological inspired routing-ACO and location information of nodes.

\section{RELATED WORK}

\section{A. Classical Routing Protocols}

Classical routing protocols are proactive, reactive and hybrid [4, 5]. Routing protocol has an impact on energy consumption behavior of nodes in a network. Since mobile nodes in the WSN are supplied with a limited energy battery, energy efficiency is a major problem that influences the overall network performance. Taking energy into consideration, reactive protocols are more efficient as compared to proactive protocols due to less control overheads. Both proactive and 
reactive protocols are unaware of energy metrics and hence cause lowering of the battery energy of the nodes over the most heavily used routes in the network.

\section{B. Localization Based Routing Protocols}

Location information for sensor networks by most routing protocols needs to calculate the distance between two particular nodes so that energy consumption can be calculated. According to the dependency of range measurements, the existing localization schemes can be categorized into two major categories: range-based approaches and range-free approaches. Range-based and range-free schemes are again divided into anchor based and anchor free schemes. The anchor-free schemes do not assume that the node positions are known at first. On the other hand, the anchor-based schemes need some nodes that are aware of their positions (anchor nodes) to provide geographic information to unidentified nodes to localize [6].

\section{Location Based Bio-Inspired Routing Algorithms}

Various location-based routing algorithms have been proposed, nevertheless, they have a relative shortcoming: either not guaranteeing to find a way to the destination or locating a path which is much longer than the shortest path. Position based ant colony (POSANT) routing algorithm is a collection of ant colony based routing algorithms that use the data about the position of nodes to improve the efficiency of an ant algorithm. Contrary to other position based algorithms, this algorithm does not fail when the network contains nodes with different transmission range. POSANT is a multipath routing algorithm using GPS to find position information which adds to cost of nodes and is not suited for indoor network [7]. Location based ant colony optimization (LOBANT) algorithm uses distance to consider routing metrics due to received signal strength indicator (RSSI), but energy aware metrics are not taken into account [8]. Autonomous localization based eligible energetic Path_with_Ant colony optimization (ALEEP with ACO) algorithm was developed in [9] by a combination of the advantages of the best exiting protocols. Authors used the location of the nodes, adaptive transmission power (ATP) and energy aware metrics to increase the efficiency of routing. After studying the related work, we came to conclusion and proposed an event based clustering localized energy efficient ant colony optimization (EBC LEE-ACO) routing algorithm by combining the advantages of ACO, RSSI and clustering.

\section{PROPOSED SCHEME}

\section{A. Problem Definition}

The main challenge of WSN is to discover efficient routing, as the sensor nodes are not static and change their position randomly. Limited battery life is another issue. A disaster situation is one more challenge for WSN by which the communications in the network may fail and lead to excessive packet drop and can hang the network. For solving these problems, EBC_LEE-ACO algorithm is proposed, which is ACO, based on geographical location with clustering approach.

\section{B. Objectives}

- To find and reconstruct the optimal path for routing in disaster situations smoothly and quickly.
- To reconstruct communication links in case of link failure.

- To reduce network energy consumption by selecting the least distance from source to destination node with localized and clustering approaches.

- To improve network QoS.

- To verify whether the proposed routing algorithm is more efficient than other routing algorithms like AODV, ACO and ACO using RSSI and present ALEEP with ACO routing algorithm.

\section{Methodology}

To achieve the objectives we considered the following implementation steps:

- Design EBC_LEE-ACO routing algorithm by combining the advantages of ACO, RSSI and clustering.

- Simulate network considering variable number of nodes and variable node mobility.

- This simulation will provide us data to perform network analysis of performance parameters like throughput, packet delivery ratio, packet drop and consumed energy.

- Compare above network parameters between the proposed algorithm and AODV, ACO, ACO using RSSI algorithm and present ALEEP with ACO algorithm.

- Network Simulator 2 (NS2) is used for simulation.

\section{CURRENT METHODS}

\section{A. Ad-hoc on Demand Distance Vector (AODV) Protocol}

As the name itself suggests, AODV protocol is an on demand routing protocol, which means that it determines a route to a destination only when a node wants to send a packet to that destination [10]. Essential objectives of the algorithm are:

- To broadcast discovery packets only when necessary using RREQ message.

- To perform local connectivity management, neighborhood identification and general topology maintenance using HELLO messages.

- To spread data about changes in availability to neighboring nodes which will probably require the information using RERR message.

AODV operation is divided into two phases, route discovery and route maintenance.

\section{B. Ant Colony Optimization (ACO) Algorithm}

ACO is a bio-inspired meta-heuristic algorithm introduced in $[11,12]$. The main idea is to use ants as an inspirational source because they follow self-organizing principles which allow highly coordinated behavior. Ants have collective learning intelligence. Each ant communicates, learns and cooperates non-verbally with the others through pheromones. Different kinds of ant's algorithm can be inspired by different ant behaviors, e.g. foraging, labor division, brood sorting, and 
cooperative transport $[13,14]$. The basic rule of ACO is the ability of ants to discover the shortest path between food sources and the anthill. In the beginning, the route the ants find may not be the shortest path, but with the passage of the time, more and more ants move cooperatively and the trail of their path becomes shorter and shorter until they get the shortest path. There are three phases of the ant based algorithm namely route discovery, route maintenance and route failure handling.

\section{Ant Colony Optimization using Received Signal Strength Indicator (ACO using RSS) Algorithm}

Location aware ACO routing is a high performance routing protocol for WSN design [15]. The main reason to seek location awareness in ACO routing is the dynamic network topology causing frequent link breakup that causes the source node to spend most of its time in route setup and route maintenance. In location awareness in ACO, each node will have a general idea about the network topology and its neighbors so that it can choose the nearest neighbor toward the destination $[15,16]$. This new routing algorithm is based on ACO and uses location as a parameter to enhance its efficiency. From the RSSI value every node can determine the distance between nodes. In ACO using RSSI, the route is searched only when there is a collection of information packets to be sent from a source node (S), to a destination node (D), thus it is a reactive routing algorithm. Sending the information packets will begin after a route from the source to the destination node is built up. Before that, only forward ants are being exchanged with backward ants. To limit the time it needs to discover a route while keeping the quantity of generated ants as small as it could be expected under the circumstances, data about the position of nodes is utilized as a heuristic value $[7,8]$. When there is a packet to be sent, the source starts a route discovery phase. At first, a route request (RREQ) broadcasts to each one of the nodes from $\mathrm{S}$ to $\mathrm{D}$. When the $\mathrm{D}$ gets the first RREQ message, its answer is a route reply message to $\mathrm{S}$. On receiving the RREP message, a node will extract RSSI value from it and would calculate the location of the neighboring nodes and in turn the location of the destination. The routing table is updated with distance information between the nodes utilizing RSSI value. Every sensor node in the WSN has a memory block in which the leftover energy, the location data of the node, its neighbors and the base station are stored. Route establishment using distance is described in $[8,20]$.

\section{PROPOSED METHODS}

\section{A. EBC_LEE-ACO Algorithm}

Previous algorithms have some limitations such as: For proper monitoring, dense and large WSN will be used for different types of applications. There is a high probability of redundant data being recorded by neighboring nodes during an event. As many nodes might sense the same event, they will establish a route separately. Routing algorithms based on ant colony are considered to have a high percentage in terms of packet delivery, but the drawback is overhead of the control messages required for discovering the route. Cluster-heads use only forward route discovery control messages. Their limitation is the dynamic topology of the network, which limits the bandwidth availability and energy constraints. To overcome these problems, clustering technique is used, as the clustering approach has an advantage of spatial reuse of resources to increase system capacity, data aggregation, reduce energy consumption etc. [17-19]. In the present research work an event based clustering localized energy efficient ant colony optimization (EBC_LEE-ACO) algorithm is proposed. The main focus of the proposed EBC_LEE-ACO algorithm is to minimize the energy consumption of the network by cluster formation and to select the optimal path based on the biological inspired routing ACO and location information of nodes.

\section{B. Three Phases (Stapes) of Proposed Algorithm}

\section{1) Hop Tree Formation Phase}

In WSN, data transmission takes place in multi-hop fashion where each node forwards its data to a neighbor node nearer to the sink. The node doesn't have a full understanding of the network, but has only knowledge of its neighboring nodes. Each node only knows the hop level in which it is in a hop tree [20]. In this phase, the distance metric used is the hop count (i.e. the number of nodes from A to B). The distances between the sink and different nodes are calculated. The algorithm is initiated by the sink node broadcasting a hop configuration message (HCM) to its neighboring nodes with a hop value. This hop value gets incremented every time the message is transmitted and is stored in their routing table. This process is continued until all the nodes are configured with a hop value within a tree.

The HCM has two parts ID and HopToTree (HTT). ID is the node identifier whereas the HTT is the distance in hops. In this approach the sink node broadcasts HCMs having values of HTT as 1 and hop count 0 . The receiving nodes forward messages to their neighboring nodes. Initially all nodes set the value of HTT as infinity. On receiving the HCM, each node compares the value of HTT in the HCM with the value of HTT that node already has in it. If the above conditions are met, then the node updates its internal stored values by the value of the field ID as well as the value of the HTT variable of the HCM. The node broadcasts the HCM with the new values. If the condition is not met which means that the node received the HCM from a shorter distance then the node discards it. The step described above occurs repeatedly until the whole network is configured. Initially there is no recognized route and the value of HTT variable has the smallest distance to the sink. When the first event is triggered the variable still stores the smallest distance, but a new route is established. After the event, the variable stores the lower among the 2 values: the distance to the sink or the distance to the closest already recognized route. Hop tree formation phase is shown in Figure 2.

\section{2) Cluster Formation and Cluster Head (CH) Selection Phase}

When an event is detected by one or more nodes, the cluster formation and the cluster head selection algorithm gets started. A set of nodes that have detected an event forms a cluster. Once clusters are formed, the next process is to select the cluster head within each cluster. The main process for forming the cluster is the selection of leader node which is called cluster head $(\mathrm{CH})$ by using the cluster configuration message (CCM). $\mathrm{CCM}$ has four attributes (Type, ID, HTT, State), where ID is 
the identifier of the node that started the CCM. HTT and state stores state value of the node.

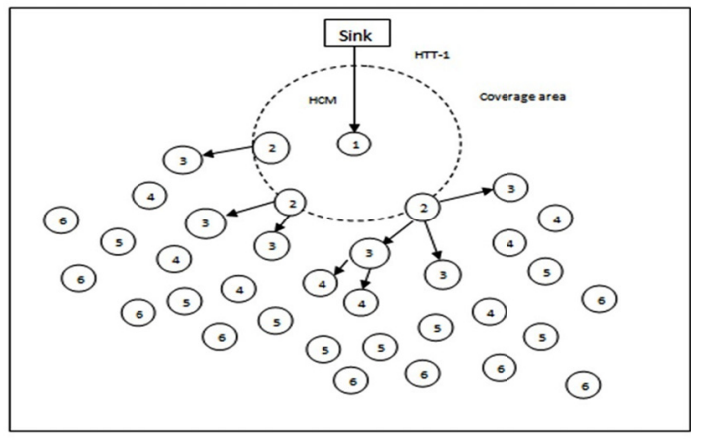

Fig. 2. Flooding of HCM message from the sink to the last node of the network where nodes are deployed randomly

If this is the first event, all sensing nodes are eligible and the cluster head is the one which is closest to either the sink node or to the node which is closest to an established route. $\mathrm{CH}$ selection is based on parameters such as hop count, energy ratio between the current energy versus the initial energy and the count that the node has been selected as $\mathrm{CH}$. In the case of a tie, i.e., the one with smaller ID wins over the others. Alternatively, one can use the energy level to select the $\mathrm{CH}$ in the case of a tie. After the end of the process, one of the nodes in the group will be selected as the $\mathrm{CH}$. The other nodes will be the members which also are a part of the same event. The $\mathrm{CH}$ collects all information gathered by member nodes and sends them to the sink. The main benefit of this algorithm is that the whole set of information gathered by various nodes sensing the same event is accumulated or aggregated at a single node, the $\mathrm{CH}$. In addition, collected information is highly correlated and therefore large amount of redundant data gets cleaned, the number of control messages for building routing tree is reduced, the number of overlapping route for efficient aggregation and reliable transmission of data is maximized. Cluster formation and $\mathrm{CH}$ selection phase of the EBC_LEEACO algorithm is shown in Figure 3.

\section{3) Route Discovery and Selection Phase}

The final step involves the route establishment through route discovery and optimal path selection for $\mathrm{S}$ (in our case coordinator) to D (sink). Actual sending and receiving of data would be done once the route is established from source to destination. Before that, only forward and backward ants are being exchanged for the route discovery process. A route is searched when the packets are required to be sent from source to a destination. The main parameter for this algorithm is the location information of the node which is used to discover the route. When the source node wants to send data to a destination node, the source node initiates a route discovery process which involves a broadcast message to be sent from source nodes to their neighboring nodes which are at 1 hop distance. With that message each node checks whether it contains the entry for the destination node in its routing table. If there is no entry the node initiates a RREQ message to its neighboring nodes which are at 1 hop distance. The process will continue until the RREQ message reaches destination node $\mathrm{D}$.

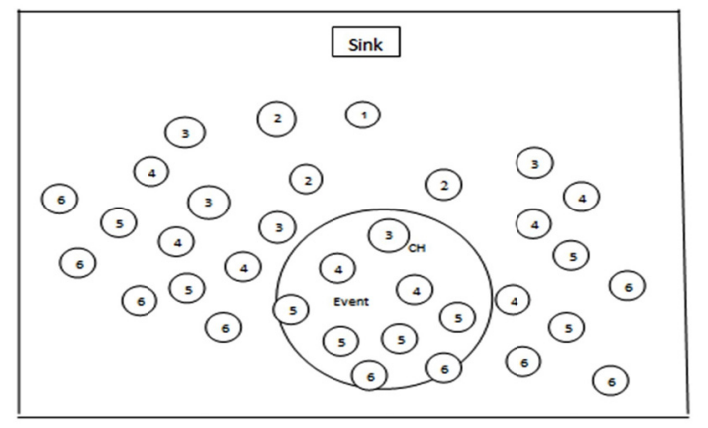

Fig. 3. Nodes detected the same event form a cluster and select $\mathrm{CH}$

If the entry of the $\mathrm{D}$ is found in any of the neighboring nodes a reply message (RREP) is generated by $\mathrm{D}$ and is sent to $\mathrm{S}$ by following back the path that was followed to reach $\mathrm{D}$. Route reply packet (RREP) contains RSSI value. When each node receives the RREP message, RSSI value would be extracted which will help in determining the location of the node and the routing table will be updated. For route selection this algorithm divides ant agents into two sections: forward ants (FANTs) and backward ants (BANTs). The main reason to divide ant agents in two sections is to take the advantage of information collected by the other section i.e. BANTs benefiting from the information collected by FANTs. Based on this principle, FANTs do not create or update routing information, but only collect the information and route creation process is done by BANTs. The process starts with the $\mathrm{CH}$ broadcasting a FANT agent towards the sink or base station to form the route for the event dissemination. The $\mathrm{S}$ initiates route establishment by sending FANTs to two possible paths from the table whose first two neighbor nodes are closer to S. The algorithm focuses on keeping the number of generated ants as low as possible and the pheromone trail shows the edge weight of the link between the nodes. These agents are communicating with each other as follows:

1. The $\mathrm{S}$ (coordinator) initiates route establishment by launching FANTs to destination at regular time intervals.

2. FANTs discover the route to D based on which node is nearer to $\mathrm{S}$ by analyzing the routing table. This process is repeated until an ant reaches $\mathrm{D}$.

3. The FANT creates a stack, pushing in trip times for every node.

4. Upon receiving a FANT each of the nodes compares its remaining energy with the threshold energy.

5. When the destination node or sink receives the first FANT, it initiates a timer called FET (forward expiration timer). During this time the D node accepts all FANTs. With received FANTs, D will calculate the optimal path using transition rule.

6. Once D is reached, FANT is converted into BANT. BANT takes the stack and follows it.

7. The BANT follows the stack entries and traces the reverse path from the sink to the source. 
8. Pheromone edge updates depend upon the residual energy of the node and location.

9. Once the optimal path is found, D backs up all possible paths in case the path is failed.

\section{PRACTICAL ANALYSIS}

This section presents a practical analysis of network performance metrics like throughput, packet delivery ratio, packet drop, consumed energy and node mobility.

\section{A. Network Scenarios and Simulation Parameters}

Scenarios and simulation parameters are shown in Table I.

TABLE I. SCENARIOS AND SIMULATION PARAMETERS

\begin{tabular}{|c|c|}
\hline $\begin{array}{c}\text { Network Parameters } \\
\text { Routing Protocol } / \\
\text { Algorithm }\end{array}$ & $\begin{array}{c}\text { AODV, ACO, ACO using } \\
\text { RSSI, EBC_LEE-ACO }\end{array}$ \\
\hline Traffic Patterns & CBR (Constant Bit Rate) \\
\hline Network Size & $1000 \times 1000(\mathrm{X} \times \mathrm{Y})$ \\
\hline MAC Protocol & 802.11 \\
\hline Initial Energy & 200J (for each node) \\
\hline Simulation Time & NS-allinone-2.32 \\
\hline Simulation Platform & $10 / 30 / 60 / 100$ \\
\hline \multicolumn{2}{|c|}{ Node Variables } \\
\hline Number of Nodes & $3 \mathrm{~m} / \mathrm{s}$ \\
\hline Node Speed & 50 \\
\hline \multicolumn{2}{|c|}{ Variable Mobility } \\
\hline Number of Nodes & $1 / 2 / 3 / 4 / 5 \mathrm{~m} / \mathrm{s}$ \\
\hline Maximum Speed &
\end{tabular}

\section{B. Results and Analysis}

\section{1) Results on Varying Number of Nodes}

It is observed from Figures 4 to 7 that for increasing number of nodes and constant mobility, throughput and packet delivery ratio decrease and consumed energy and the number of dropped packets increase because:

- the probability of success in accessing the channel decreases,

- as hop count increases, congestion and delay increases and collision and transmission error increase.

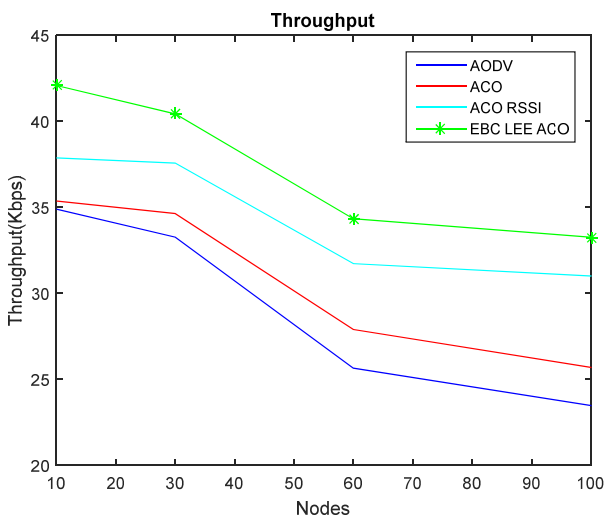

Fig. 4. Throughput versus number of nodes

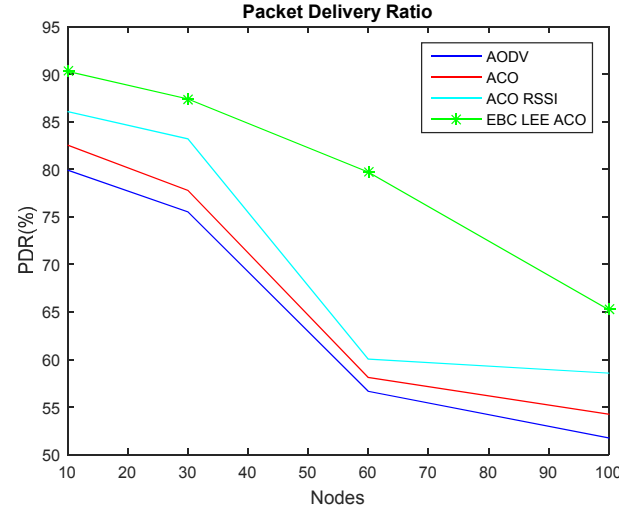

Fig. 5. PDR versus number of nodes

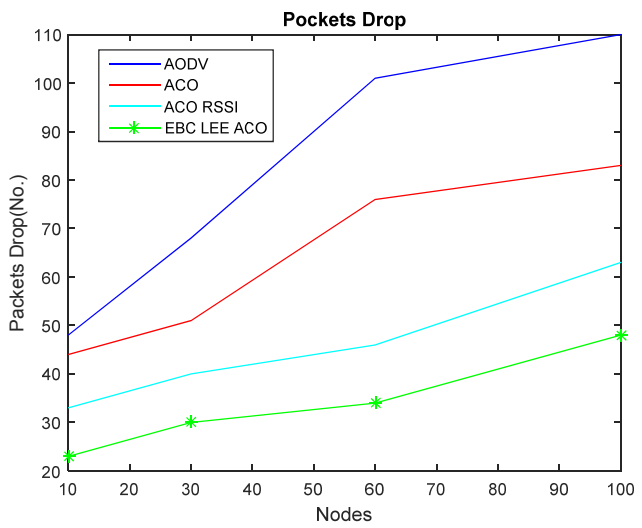

Fig. 6. Packets drop versus number of nodes

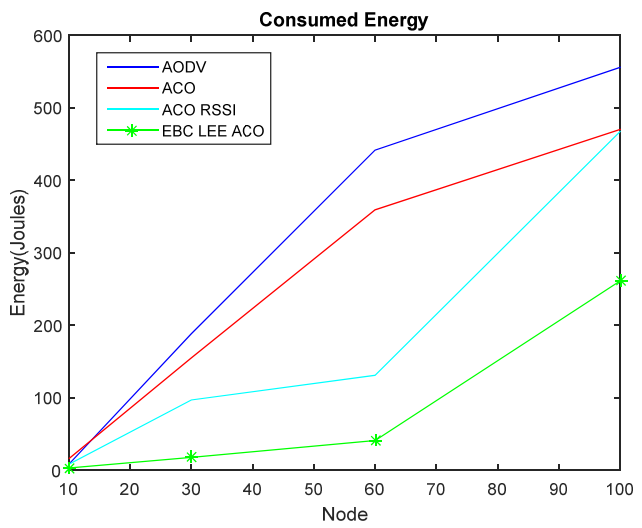

Fig. 7. Energy versus number of nodes

2) Results of Varying Node Mobility

Figures 8 to 11 show that for increasing mobility with constant nodes, throughput, packet delivery ratio and consumed energy decrease and packet drop increases because the probability of path breakage increases and the construction of a new path takes time. Results show that the performance parameters of the network are improved by the use of the proposed EBC LEE-ACO algorithm in comparison with AODV, ACO and ACO using RSSI algorithms due to the following characteristics of the EBC_LEE-ACO algorithm: 
- No back propagation.

- Multipath routing.

- No packet flooding.

- Shortest distance routing.

- Ideal nodes.

- Reduce overhead.

- Data aggregation.

- No redundant data transmission.

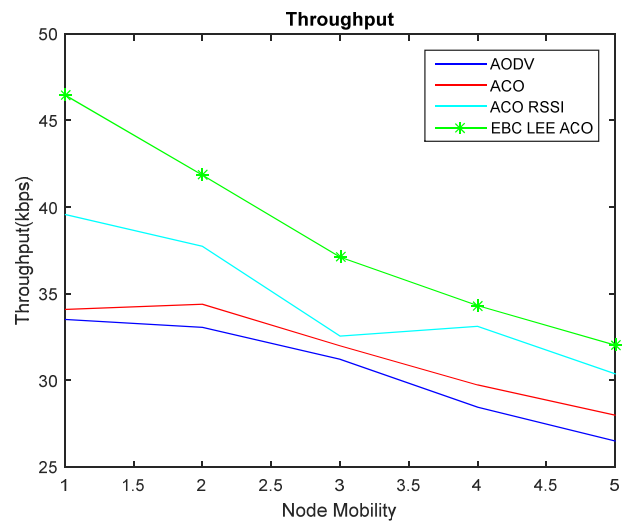

Fig. 8. Throughput versus node mobility

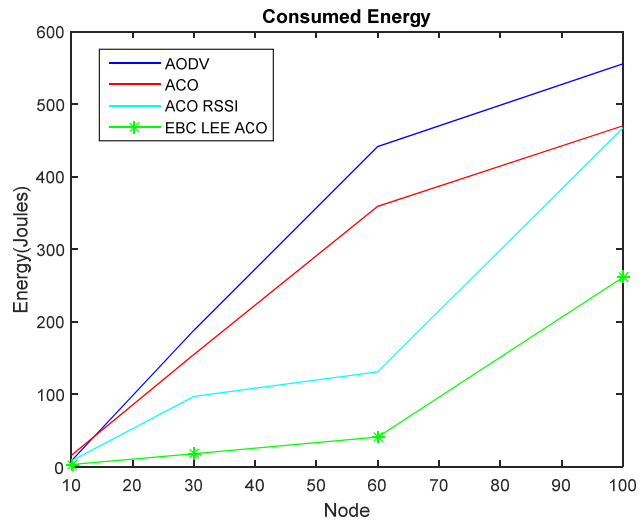

Fig. 9. Energy versus number of nodes

\section{CONCLUSIONS}

In this work, the EBC_LEE-ACO routing algorithm is implemented and the network performance, by varying number of nodes and node mobility, is analyzed. This algorithm is extensively compared to other algorithms like AODV, ACO and ACO-RSSI by considering network metric parameters like throughput, packet delivery ratio, packet drop and consumed energy. Simulation results show that the EBC_LEE-ACO algorithm outperformed the other algorithms in disaster situations. ACO achieves better performance compared to
AODV, as ACO allows rerouting to another link in the case of existing link failure (no back propagation). ACO using RSSI routing algorithm improves the routing by minimizing the flooding of routing packets because, it has the location information of nearby nodes.

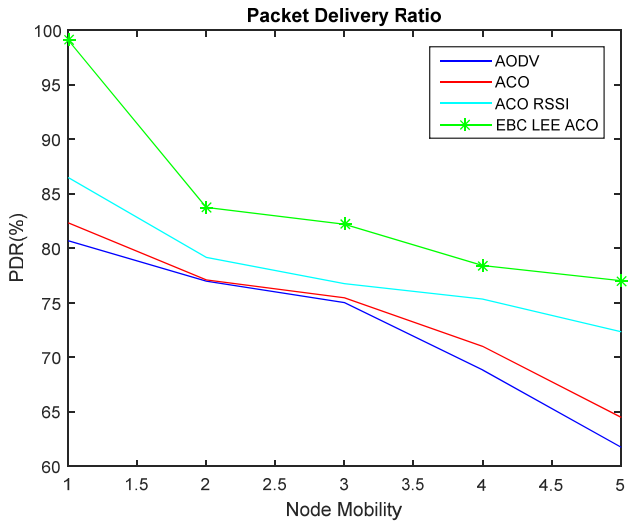

Fig. 10. PDR versus node mobility

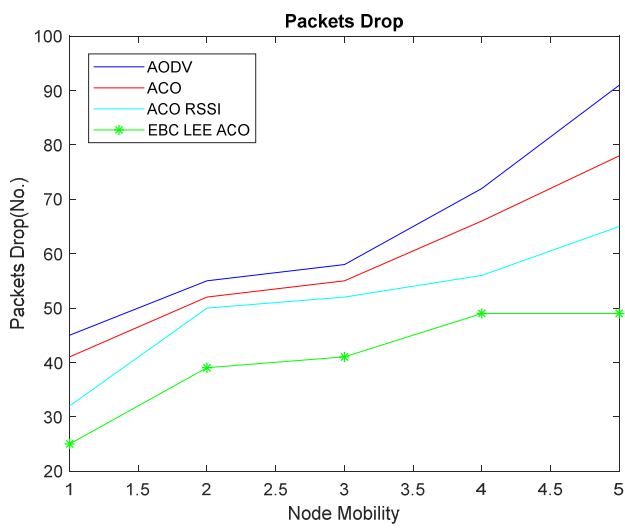

Fig. 11. Packet drop versus node mobility

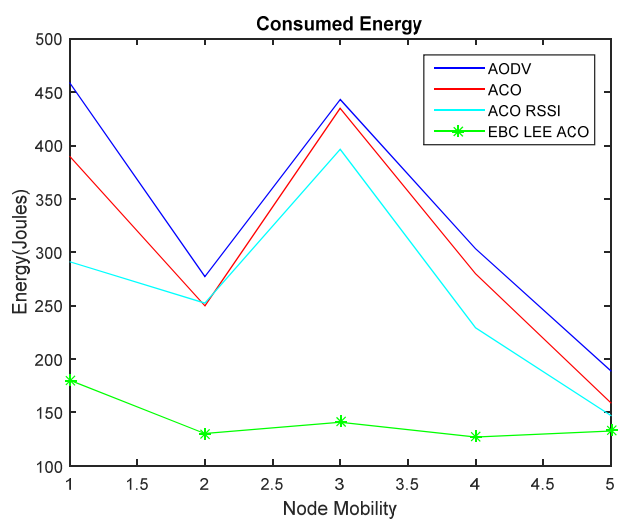

Fig. 12. Energy versus node mobility

The EBC_LEE-ACO algorithm has achieved better performance due to clustering technique and location information of nodes. Clustering data is aggregated and sent to the sink through $\mathrm{CH}$ which reduces overheads. Also, location 
information of nodes is useful to send data to shortest distance node in less time. The proposed algorithm reduces energy consumption by approximately 7\%. An improvement in throughput, packet delivery ratio and increase in packet drop has been observed in comparison with present network routing algorithms, i.e. autonomous localization based eligible energetic Path_with_Ant colony optimization (ALEEP with ACO) [9]. Use of IEEE 802.11 standard increased packet drop. Hence, our EBC_LEE-ACO algorithm is useful for improvement in QoS and reduction in energy consumption of the WSN. It is most suitable for information monitoring in disaster situations. The extension of the proposed algorithm will be considered for varying network areas as well as increasing number of nodes and mobility in future work.

\section{REFERENCES}

[1] I. F. Akyildiz, W. Su, Y. Sankarasubramaniam, E. Cayirci, "Wireless sensor networks: a survey", Computer Networks, Vol. 38, No. 4, pp. 393-422, 2002

[2] J. Yick, B. Mukherjee, D. Ghosal, "Wireless sensor network survey", Computer Networks, Vol. 52, No. 12, pp. 2292-2330, 2008

[3] S. K. Gupta, P. Sinha, "Overview of Wireless Sensor Network: A Survey", International Journal of Advanced Research in Computer and Communication Engineering, Vol. 3, No. 1, pp. 5201-5207, 2014

[4] A. K. Gupta, H. Sadawarti, A. K. Verma, "Review of Various Routing Protocols for MANETs", International Journal of Information and Electronics Engineering, Vol. 1, No. 3, pp. 251-259, 2011

[5] H. S. A. Hamatta, N. I. Zanoon, R. M. Al-Tarawneh, "Comparative Review for Routing Protocols in Mobile Ad-Hoc Networks", International Journal of Ad hoc, Sensor \& Ubiquitous Computing, Vol. 7, No. 2, pp. 13-31, 2016

[6] A. Mesmoudi, M. Feham, N. Labraoui, "Wireless Sensor Networks Localization Algorithms: A Comprehensive Survey", International Journal of Computer Networks \& Communications, Vol. 5, No. 6, pp. 45-64, 2013

[7] S. Kamali, J. Opatrny, "A Position Based Ant Colony Routing Algorithm for Mobile Ad-hoc Networks", Third International Conference on Wireless and Mobile Communications (ICWMC'07), Guadeloupe, France, March 4-9, 2007

[8] R. Vallikannu, S. E. Jubin, “A Location Based ACO Routing Algorithm For Mobile Ad Hoc Networks Using RSSI", IEEE International Conference on Communication and Signal Processing, Chennai, India, April, 3-5, 2013

[9] R. Vallikannu, A. George, S. K. Srivatsa, "Autonomous localization based energy saving mechanism in indoor MANETs using ACO", Journal of Discrete Algorithms, Vol. 33, pp. 19-30, 2015

[10] P. K. Maurya, G. Sharma, V. Sahu, A. Roberts, M. Srivastava, "An Overview of AODV Routing Protocol", International Journal of Modern Engineering Research, Vol. 2, No. 3, pp. 728-732, 2012

[11] M. Dorigo, C. Blum, "Ant colony optimization theory: A survey", Theoretical Computer Science, Vol. 344, No. 2-3, pp. 243-278, 2005

[12] C. Blum, "Ant colony optimization: Introduction and recent trends", Physics of Life Reviews, Vol. 2, No. 4, pp. 353-373, 2005

[13] S. Binitha, S. S. Sathya, "A Survey of Bio inspired Optimization Algorithms", International Journal of Soft Computing and Engineering, Vol. 2, No. 2, pp. 137-151, 2012

[14] O. Deepa, A. Senthilkumar, "Swarm Intelligence from Natural to Artificial Systems: Ant Colony Optimization", International Journal on Applications of Graph Theory in Wireless Ad hoc Networks and Sensor Networks, Vol. 8, No.1, pp. 9-17, 2016

[15] X. Wang, Q. Li, N. Xiong, Y. Pan, "Ant Colony Optimization-Based Location-Aware Routing for Wireless Sensor Networks", in: Lecture Notes in Computer Science, Vol. 5258, pp. 109-120, Springer, 2008

[16] C. Dominguez-Medina, N. Cruz-Cortes, "Energy-Efficient and LocationAware Ant Colony Based Routing Algorithms for Wireless Sensor
Networks", 13th Annual Conference on Genetic and Evolutionary Computation, Dublin, Ireland, pp. 117-124, July 12-16, 2011

[17] S. K. Popat, M. Emmanuel., "Review and Comparative Study of Clustering Techniques", International Journal of Computer Science and Information Technologies, Vol. 5, No. 1, pp. 805-812, 2014

[18] S. Mahajan, P. K. Dhiman, "Clustering in Wireless Sensor Networks: A Review", International Journal of Advanced Research in Computer Science, Vol. 7, No. 3, pp. 198-201, 2016

[19] S. K. Gupta, N. Jain, P. Sinha, "Clustering Protocols in Wireless Sensor Networks: A Survey", International Journal of Applied Information Systems, Vol. 5, No. 2, pp. 41-50, 2013

[20] L. Aparecido Villas, A. Boukerche, H. Soares Ramos, H. A. B. Fernandes de Oliveira, R. Borges de Araujo, A. A. Ferreira Loureiro, "DRINA: A Lightweight and Reliable Routing Approach for in-Network Aggregation in Wireless Sensor Networks", IEEE Transactions on Computers, Vol. 62, No. 4, pp. 676-689, 2013 